\title{
PRESENT STATUS OF J-PARC RING RF SYSTEMS
}

\author{
M. Yoshii", S. Anami, E. Ezura, K. Hara, Y. Hashimoto, C. Ohmori, A. Takagi, M. Toda KEK, \\ Tsukuba, 305-0801, Japan \\ K. Haga, K. Hasegawa, M. Nomura, A. Schnase, F. Tamura, M. Yamamoto JAEA, Tokai, Japan
}

\section{Abstract}

The rapid cycling synchrotron (RCS) radio frequency accelerating systems have been prepared for the beam commissioning scheduled in the middle of September 2007. Installations of cavities, power supplies and amplifiers were carried out. And, the systems have been checked for the combined operations. For the main synchrotron (50GeV-MR) accelerating systems, the high power examinations and final adjustments are ongoing. And, installations are planned in September 2007. Here, we report on various issues that had been found and solved during the examination and installation period.

\section{INTRODUCTION}

The beam commissioning of the J-PARC linac started December 2006 and the proton beam was successfully accelerated to the design energy of $181 \mathrm{MeV}$. Recently, the construction of RCS has reached the most demanding period. Installations and post-examinations of each component were started. The mass-measurements of the magnetic alloy cores have been scheduled and executed for both RCS and MR RF systems. The 1000-hours long run high power test was carried out to prove the reliability of the core production. Then, the remaining cores were tested for 300 hours. The testing of RCS cores for ten cavities (180 cores in total) was successfully finished in April 2007. All cavities and final stage tube amplifiers were installed in the RCS tunnel (Figure 4). The auxiliary DC power supplies and driver amplifiers are put into the designated positions. And, the production and testing of the MR cores for five cavities (90 cut-cores) will be finished by the end of July 2007. Installation of MR RF systems is planned to start in this August after the high power test are done.

\section{RF SYSTEMS}

The cavity provides a high field gradient of $\sim 25 \mathrm{kV} / \mathrm{m}$ in both RCS and MR synchrotrons. The designed accelerating voltage is $45 \mathrm{kV} /$ cavity. Magnetic alloy (MA) materials are employed for loading the cavity, instead of conventional ferrite materials. MA is a material that is stable under high RF magnetic field. The frequency range of the RCS system is $0.94 \mathrm{MHz}-1.67 \mathrm{MHz}$ for $\mathrm{h}=2$. The optimum Q-value for the RCS system is $\mathrm{Q}=2$ [1]. The combined RF signals of a fundamental $(\mathrm{h}=2)$ and the second harmonic $(\mathrm{h}=4)$ are combined and fed into the same cavity. The frequency range of MR system is $1.67 \mathrm{MHz}-1.72 \mathrm{MHz}$ and the frequency variation is only $3 \%$. The harmonics for acceleration $(\mathrm{h}=9)$ and bunch

*masahito.yoshii@kek.jp shaping $(\mathrm{h}=18)$ are fed to separate cavities in case of the MR RF system. The optimum Q-value becomes 10-20 when considering transient beam loading.

The Q-value of un-cut cores is 0.6. In order to adjust the Q-value of the systems, several configurations are considered to meet the requirements.

\section{Realizing $Q=2$ RCS Cavity}

The cut-core configuration was the original idea to realize $\mathrm{Q}=2$. However, the practical distance between two halves of cut-core became $<1 \mathrm{~mm}$. This is almost comparable to the machining tolerance. Also, it was an issue how to guide the cooling water in such a narrow region. The "Hybrid-cell" configuration was considered to solve the technical difficulties with the original cutcore idea. This cavity consists of two uncut core cells $(\mathrm{Q}=0.6)$ and one cut core cell $(\mathrm{Q}=4)$, which are combined in this configuration [2]. The distance between each two core-halves is about $1.5 \mathrm{~mm}$, which is more relaxed than the original distance. Another configuration using a parallel inductor and vacuum capacitor has been proposed to adjust the Q-value and the resonance of the uncut core loaded cavity. In this case, the production process can be considerably abridged and reliability is improved.

The equivalent circuit of a 3-gap system with un-cut cores is shown in Figure 1(a). By adding the lossless inductor and capacitor externally in parallel (Figure 1(b)) the Q-value can be adjusted following the relation $\mathrm{Q}=\mathrm{R} 1 / \omega \mathrm{L} 3$.
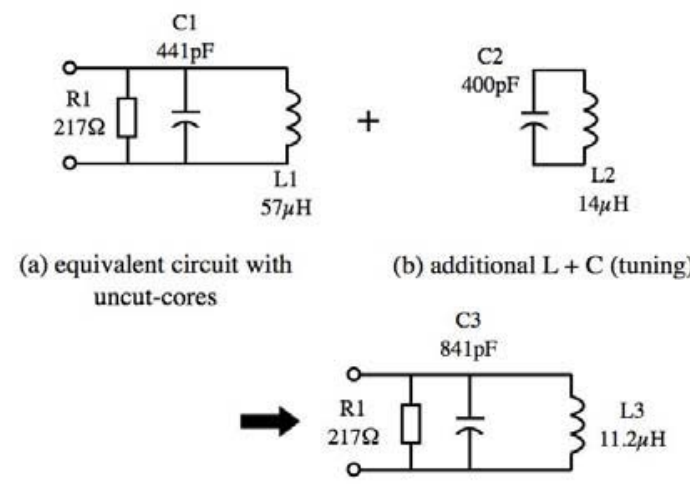

(c) required $Q=2$ circuit

Figure 1: "Parallel Inductance" Configuration 
The "Hybrid-cell" and "Parallel inductance" setups are tested. And, we confirmed that both configurations can realize the optimum $Q=2$. Finally, we adopted the "Parallel Inductance" configuration for RCS RF systems (Figure 1(c)) [3].

The magnetic alloy cores are made by first winding and later annealing the thin insulating amorphous ribbon. The thickness of insulation is typically $2 \mu \mathrm{m}$. The waterproofcoated MA cores are set inside the water-filled cavity tanks. The cores are directly cooled by dissolve-oxygen free de-mineralized water. Since the dielectric constant of water is high, RF coupling between the cores and the water tank becomes strong. The radial radio-frequency impedance of toroidal core has to be taken into account carefully in the process of shuffling to define the locations of the cores inside the tank. It was an issue to produce flawless cores during the winding process and the postprocess coating [4].

\section{Cut-cores for $50 \mathrm{GeV}$ MR RF Cavity}

The optimum Q-value for beam acceleration in the $50 \mathrm{GeV}$ ring is $10-20$. In this case, the functions for acceleration and bunch manipulation have to be separated. Indeed, seven spaces are for the acceleration systems and another four spaces are reserved for $2^{\text {nd }}$ harmonics systems. In order to realize the medium $\mathrm{Q}$ radiofrequency cavity systems, the cut core configuration is necessary in case of the MR system [5]. The "Parallel Inductance" configuration is not feasible for the MR system, because the parallel inductor L2 (in Figure 1(b)) becomes too small, and the losses are increased. Also, there is the problem that uncut cores with MR dimension can overheat near the inner radius. The cut core production includes several key process-steps as follow, (a) immersion with low viscous epoxy, (b) waterproof- coating, (c) water-jet cutting, (d) $2^{\text {nd }}$ immersion and (e) diamond polishing. The first immersion process is important to keep the mechanical strength during the water-jet cutting process and $2^{\text {nd }}$ immersion is for the diamond polishing process. The diamond polishing is a necessary process in order to recover the electrical isolation along the cut-surface where the isolation is damaged during the water-jet process. The diamond polishing took the place of grinding stone cutting + etching [6]. Figure 2 compares the surfaces obtained in two different ways. The cut surface finished by diamond polishing features a mirror like quality. The surfaces are too smooth for existing epoxy coating. The polished cutcores were examined during a 1000-hour high power test with high-purity de-mineralized plus low dissolved oxygen $(<10 \mathrm{ppb})$ water. The cut-surfaces were covered by stable magnetite. This black coating seems to protect against further corrosion.

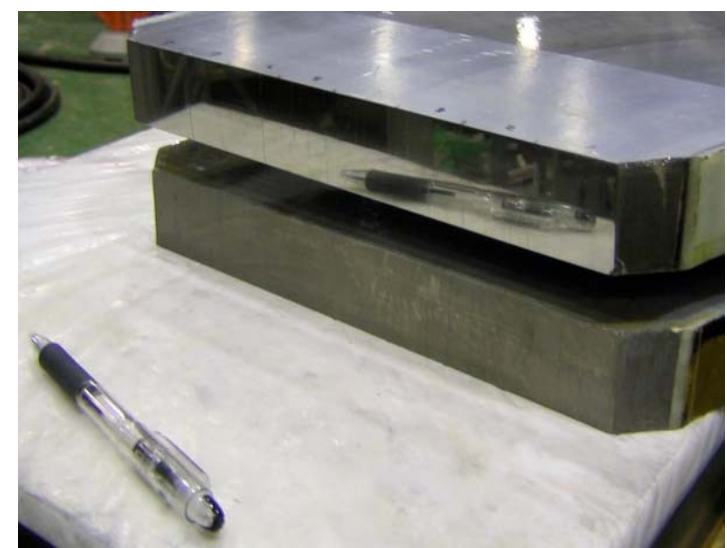

Figure 2: Cut cores finished by "Diamond Polishing" (upper) and "Grinding Stone" (lower)

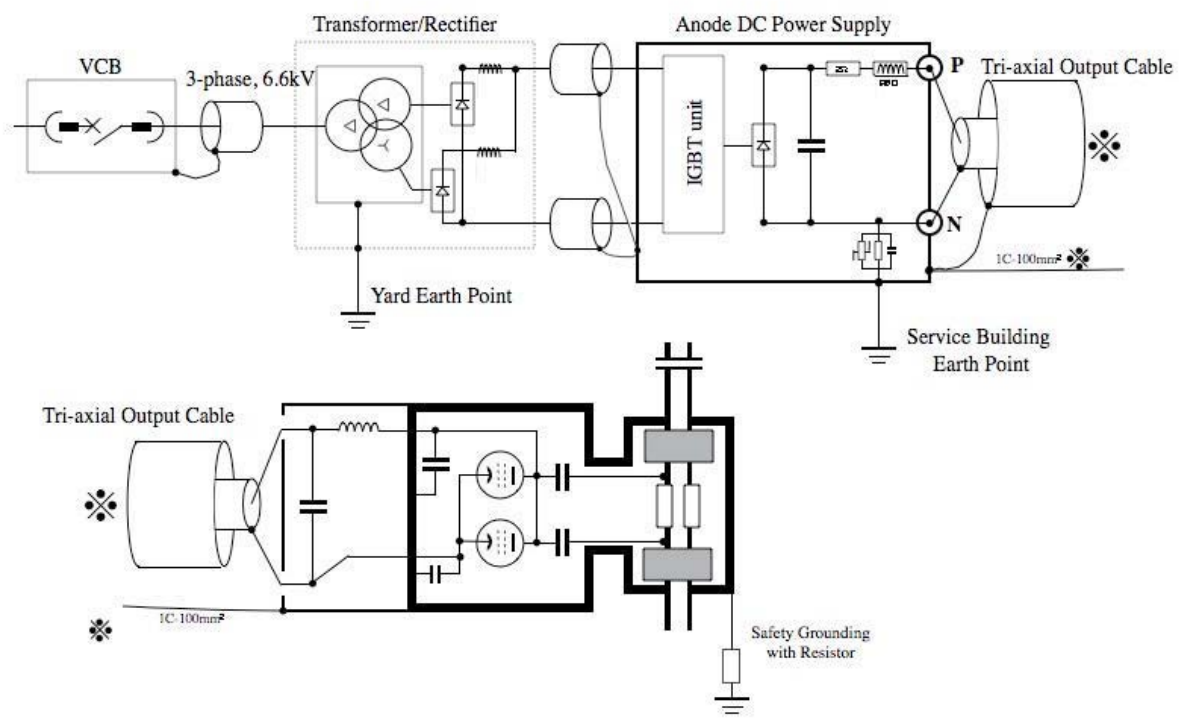

Figure 3: Grounding of Anode power supply-cavity system 


\section{Grounding and Power Distribution of the Systems}

We must pay careful attention to the grounding and power distribution of each system. Especially, IGBT based switching mode anode power supplies produce radio frequency noises in the order of several hundred $\mathrm{kHz}$ to a few $\mathrm{MHz}$, which overlap the accelerating radio frequency. The synchrotron frequency bands are $6 \mathrm{kHz} \sim$ $400 \mathrm{~Hz}$ in RCS and $600 \mathrm{~Hz} \sim 20 \mathrm{~Hz}$ in MR. The IGBT inverter anode DC power supply uses high switching frequency around $30 \mathrm{kHz}$ and the output units are connected in parallel [7]. The equivalent switching frequency is $468 \mathrm{kHz}$ at maximum and well separated from the synchrotron frequency. To realize a low plate voltage ripple $(<0.2 \%)$, voltage feedback control loop and current feed-forward loop are included. A $100 \mu \mathrm{H} \mathrm{RF}$ choke coil at the output circuit of the power supply and a pi-type filter on an input circuit of the tube amplifier minimize radio frequency noises.

All power supply-cavity systems are electrically isolated from ground. For safety reason, each cavity must be connected to ground with a series resistor. The output cable of each anode power supply is a tri-axial cable (Figure 3). The inner conductor is connected to the positive and the middle conductor is connected to the negative output of the anode power supply. And, the third (outer) conductor foreseen to shield the noises and connected to either side of the power supply or the tube amplifier. It is important that the power distribution is designed so that the anode currents do return to the power supply only through the proper return path.

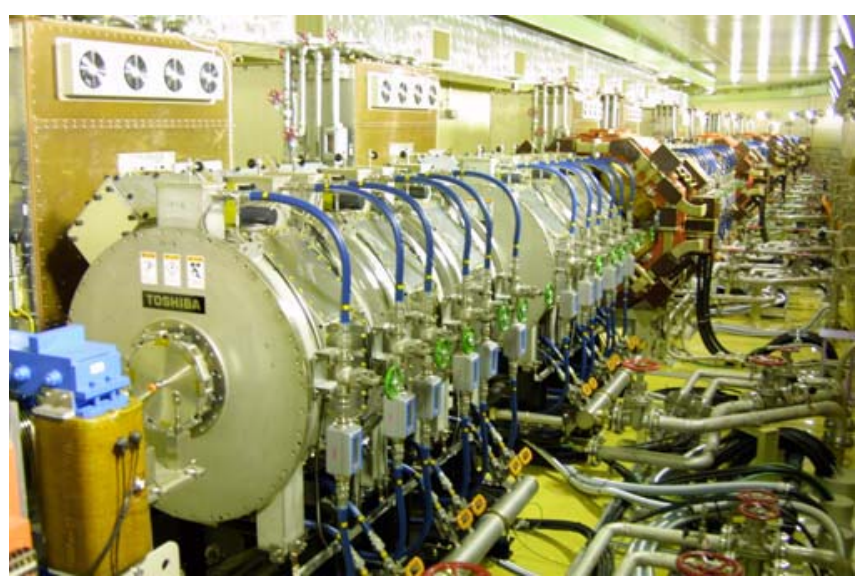

Figure 4: Ten RF systems installed in the RCS tunnel.

\section{SUMMARY}

The RF system has the two major functions of accelerating the beam and manipulating the beam distribution. The RCS RF system combines both functions in each cavity. We have studied 3 different configurations to realize the $\mathrm{Q}=2 \mathrm{RCS}$ cavity; i.e. the "Cut-core", "Hybrid-cell" and "Parallel Inductance". Finally, the
"Parallel Inductance" configuration has been chosen, because of reliability and reduced number of manufacturing processes compared to the other alternatives. The relation between layer insulation and $\mathrm{RF}$ impedance of MA cores was carefully investigated and gave valuable feedback to the production processes. All RCS cores are measured/ tested and shuffled. This mass measurement started last May 2006, and was completed in April 2007. The RCS 10 RF systems are ready and the installation is finished in the end of June 2007. Figure 4 shows the cavities and adjacent amplifiers located in the RCS tunnel. The beam commission in RCS is scheduled in the middle of September 2007. The system operation by central remote control is going to start in July. Grounding and power distribution of the system are laid out carefully so as not to adversely affect the beam or the control due to the MW-class IGBT switching mode power supplies. Electrical isolation from "ground" lines is a key concern to eliminate ground loops.

The MR RF system has separated cavities for acceleration and beam shaping. In view of narrow frequency sweep and the transient beam loading we choose a higher Q system, with Q around 20. The diamond polishing method was developed to product high-quality cut-cores. The cut surfaces are extremely smooth and have a mirror like quality. The flatness of a Cshape cut core is $\sim 100 \mu \mathrm{m}$ or better. The cut cores have a waterproof coating except at the cut-surfaces. The high power long runs have been carried out and will be completed by the end of July 2007. Installation of the MR RF systems will start in August.

\section{REFERENCES}

[1] M. Yoshii, et.al, "Present Status of J-PARC Ring RF Systems", PAC05, Knoxville, Tennessee, USA, p. 475

[2] A. Schnase et.al, "Hybrid Cavity for J-PARC Rapid Cycling Synchrotron", Proceedings of $15^{\text {th }}$ symposium on Accelerator Science and Technology, 2005, Japan

[3] A. Schnase et.al, "MA Cavities for J-PARC with Controlled Q-value by External Inductor", PAC07, Albuquerque, USA

[4] M. Yamamoto et.al, "High Power Test of MA Cavity for J-PARC RCS", PAC07, Albuquerque, USA

[5] T. Uesugi et.al, "Direct-cooling MA Cavity for JPARC Synchrotrons", PAC03, Portland, Oregon, USA, P.1234

[6] C. Ohmori et.al, "New Cutting Scheme of Magnetic Alloy Cores for J-PARC Synchrotrons", page 1313, EPAC06, Edinburgh, Scotland

[7] M. Souda et.al, "Development of High-power Highvoltage DC Power Supply Based on Series Resonant Technology for MW Class RF Amplifier", Proceedings of $14^{\text {th }}$ symposium on Accelerator Science and Technology, 2003, Japan

A15 High Intensity Accelerators 